\title{
Hidden variable models for quantum theory cannot have any local part
}

\author{
Roger Colbeck and Renato Renner \\ Institute for Theoretical Physics \\ ETH Zurich, Switzerland \\ colbeck@phys.ethz.ch renner@phys.ethz.ch
}

\begin{abstract}
It was shown by Bell that no local hidden variable model is compatible with quantum mechanics. If, instead, one permits the hidden variables to be entirely non-local, then any quantum mechanical predictions can be recovered. In this paper, we consider general hidden variable models which can have both local and non-local parts. We then show the existence of (experimentally verifiable) quantum correlations that are incompatible with any hidden variable model having a non-trivial local part, such as the model proposed by Leggett.
\end{abstract}

\section{INTRODUCTION}

Consider a source emitting two particles, which travel to two detectors, located far apart. The detectors are controlled by Alice and Bob. We denote Alice's choice of measurement by $A$, and similarly Bob's by $B$. The measurement devices generate the outcomes $X$ and $Y$ on Alice's and Bob's sides, respectively.

In a hidden variable model, one attempts to describe the outcomes of such measurements by assuming that there are hidden random variables, in the following denoted by $U, V$, and $W$, distributed according to some joint probability distribution $P_{U V W}$. (For reasons to be clarified below, we consider three different hidden variables.) Measurement outcomes then only depend on Alice and Bob's choice of measurement $A$ and $B$ as well as the values of the hidden variables $U, V, W$, that is, formally

$$
\begin{aligned}
X & =f(A, B, U, V, W) \\
Y & =g(A, B, U, V, W)
\end{aligned}
$$

for some functions $f$ and $g$. Rephrased in the language of conditional probability distributions, these conditions read

$$
\begin{aligned}
& P_{X \mid A=a, B=b, U=u, V=v, W=w}(x)=\delta_{x, f(a, b, u, v, w)} \\
& P_{Y \mid A=a, B=b, U=u, V=v, W=w}(y)=\delta_{y, g(a, b, u, v, w)} .
\end{aligned}
$$

In this work, we divide the hidden variables into local and non-local parts: ${ }^{1} U$ and $V$ are, respectively, Alice's and Bob's local hidden variables, and $W$ is a non-local hidden variable. The requirement is that, when the non-local part $W$ is ignored, Alice's distribution depends only on the local parameters $A, U$ and Bob's only on $B, V$,

$$
\begin{aligned}
& \sum_{w} P_{W}(w) P_{X \mid A=a, B=b, U=u, V=v, W=w} \equiv P_{X \mid A=a, B=b, U=u, V=v} \equiv P_{X \mid A=a, U=u} \\
& \sum_{w} P_{W}(w) P_{Y \mid A=a, B=b, U=u, V=v, W=w} \equiv P_{Y \mid A=a, B=b, U=u, V=v} \equiv P_{Y \mid B=b, V=v} .
\end{aligned}
$$

We stress here that identities (11) and (2) do not restrict the generality of the model; they are merely a definition of what we call local. In fact, any possible dependence of the individual measurement outcomes $X$ and $Y$ on the choice of measurements $A$ and $B$-in particular, the predictions of quantum theory - can be recreated by an appropriate choice of functions $f$ and $g$ that depend on the non-local variable $W$ but not on the local variables $U$ and $V$. In the following, we call such a model entirely non-local. The de Broglie-Bohm theory (see, e.g., [2]) is an example of such a model.

In the Bell model [2], one makes the assumption that the individual measurement outcomes are fully determined by local parameters, i.e., that the functions $f$ and $g$ only depend on the local variables $U$ and $V$, respectively,

\footnotetext{
${ }^{1}$ Notice that our definition of local and non-local parts is not the same as that used in [1]. While ours is based on a distinction between local and non-local hidden variables, the definition in [1] relies on a convex decomposition of the conditional probability distribution into a local conditional distribution and a non-local one.
} 
but not on $W$. It is well known that such an assumption is inconsistent with quantum theory. Modulo a few loopholes (see for example [3, 4, 5] for discussions), experiment agrees with the predictions of quantum mechanics, and hence falsifies Bell's model.

Leggett [6] has introduced a hidden variable model for which the hidden variables have both a local and a global part as above. In addition, he assumes that the expectation values of the measurement outcomes obey a specific law (Malus' law), which depends only on local quantities. More concretely, the assumption is that the measurement outcomes $X$ and $Y$ are binary values and that the measurement choices $A=\vec{A}$ and $B=\vec{B}$ as well as the local hidden variables $U=\vec{U}$ and $V=\vec{V}$ are unit vectors. The conditional probability distributions $P_{X \mid A=a, U=u}$ and $P_{Y \mid B=b, V=v}$ on the r.h.s. of (1) and (2) are given by $\left[|\vec{A} \cdot \vec{U}|^{2}, 1-|\vec{A} \cdot \vec{U}|^{2}\right]$ and $\left[|\vec{B} \cdot \vec{V}|^{2}, 1-|\vec{B} \cdot \vec{V}|^{2}\right]$, respectively. Such a model is inconsistent with quantum theory, and has motivated recent experiments [7, 8, 9].

In this paper, we show that there exist quantum correlations for which all hidden variable models with a non-trivial local part are inconsistent. More precisely, we show that there is a Bell-type experiment with binary outcomes $^{2} X$ and $Y$ such that $P_{X \mid A=a, U=u}=\mathcal{U}_{X}$ for all $a$ and $u$, and $P_{Y \mid B=b, V=v}=\mathcal{U}_{Y}$ for all $b$ and $v$ are the only distributions compatible with quantum mechanics, where $\mathcal{U}_{X}$ and $\mathcal{U}_{Y}$ denote the uniform distributions on $X$ and $Y$, respectively. In particular, $X$ and $Y$ are independent of the local hidden variables $U$ and $V$. Thus, the only hidden variable model compatible with quantum mechanical predictions is entirely non-local. This is in agreement with a similar result obtained independently by Branciard et al. [10], where it is shown that Leggett-type inequalities have no local part.

\section{DEFINITIONS AND USEFUL LEMMAS}

Our technical theorem will rely on the notion of non-signaling distributions. Intuitively, a conditional distribution $P_{X Y \mid A B}$ is non-signaling if the behavior on Bob's side, specified by $B$ and $Y$, cannot be influenced by Alice's choice of $A$, and vice versa. We give a general definition for $n$ parties.

Definition 1. An $n$ party conditional probability distribution $P_{X_{1}, \ldots, X_{n} \mid A_{1}, \ldots, A_{n}}$ is non-signaling if, for all subsets $S \subseteq\{1, \ldots, n\}$, we have

$$
P_{X_{S_{1}}, \ldots, X_{S_{|S|}} \mid A_{1}, \ldots, A_{n}}=P_{X_{S_{1}}, \ldots, X_{S_{|S|}} \mid A_{S_{1}}, \ldots, A_{S_{|S|}}} .
$$

In the following, we denote by $D\left(P_{X}, Q_{X}\right)$ the statistical distance between two probability distributions $P_{X}$ and $Q_{X}$, defined by $D\left(P_{X}, Q_{X}\right)=\frac{1}{2} \sum_{x}\left|P_{X}(x)-Q_{X}(x)\right|$. It is easy to verify that

$$
D\left(P_{X}, Q_{X}\right)=\sum_{x} \max \left[0, Q_{X}(x)-P_{X}(x)\right] .
$$

Furthermore, taking marginals cannot increase the statistical distance, i.e.,

$$
D\left(P_{X}, Q_{X}\right) \leq D\left(P_{X Z}, Q_{X Z}\right)
$$

where $P_{X}$ and $Q_{X}$ are the marginals of joint distributions $P_{X Z}$ and $Q_{X Z}$, respectively. In fact, if the marginals $P_{Z}$ and $Q_{Z}$ are equal, then the distance $D\left(P_{X Z}, Q_{X Z}\right)$ can be written as the expectation of the distance between the corresponding conditional probability distributions,

$$
D\left(P_{X Z}, Q_{X Z}\right)=\sum_{z} P_{Z}(z) D\left(P_{X \mid Z=z} Q_{X \mid Z=z}\right) .
$$

Finally, we will use the following lemma which relates the statistical distance to the probability that two random variables take the same value.

Lemma 1. Given a joint probability distribution $P_{X Y}$, the distance between the marginals $P_{X}$ and $P_{Y}$ is upper bounded by the probability that $X \neq Y$, that is, $D\left(P_{X}, P_{Y}\right) \leq \sum_{x \neq y} P_{X Y}(x, y)$.

Proof. Define $X^{\prime}$ as a copy of $X$, so that $P_{X X^{\prime}}(x, y)=0$ for all $x \neq y$. Using (41) and (3), we have

$$
D\left(P_{X}, P_{Y}\right) \leq D\left(P_{X X^{\prime}}, P_{X Y}\right)=\sum_{x \neq y} P_{X Y}(x, y) .
$$

\footnotetext{
2 Note that the labeling of the outcomes $X$ and $Y$ is irrelevant for the argument. For concreteness, one might think of $X \in\{-1,1\}$ or $X \in\{0,1\}$.
} 


\section{CHAINED BELL INEQUALITIES}

We use the family of Bell inequalities introduced by Pearle [11] and Braunstein and Caves [12]. Each member of this family is indexed by $N \in \mathbb{N}$, the number of measurement choices. Alice can choose the measurements $A \in\{0,2, \ldots, 2 N-2\}$ and Bob $B \in\{1,3, \ldots, 2 N-1\}$. Each measurement has two outcomes, i.e., $X$ and $Y$ are binary. If $x$ is one outcome, $\bar{x}$ denotes the other.

The quantity we consider is

$$
I_{N} \equiv I_{N}\left(P_{X Y \mid A B}\right):=\sum_{\substack{a, b \\|a-b|=1}} \sum_{x} P_{X Y \mid A=a, B=b}(x, \bar{x})+\sum_{x} P_{X Y \mid A=0, B=2 N-1}(x, x) .
$$

Note that, for any fixed $a, b$, the sum $\sum_{x} P_{X Y \mid A=a, B=b}(x, \bar{x})$ corresponds to the probability that the values $X$ and $Y$ are distinct. It is easy to verify (but we are not going to use this fact) that all classical correlations satisfy $I_{N} \geq 1$ (i.e., $I_{N} \geq 1$ is a Bell inequality), and that the CHSH inequality [13] is the $N=2$ version. We also emphasize that the bound $I_{N} \geq 1$ is independent of the actual measurements chosen and hence allows a device-independent falsification of hidden variable models (in contrast to Leggett-type inequalities).

Using a quantum mechanical setup, one can obtain a value of $I_{N}$, denoted $I_{N}^{\mathrm{QM}}$, which is arbitrarily small in the large $N$ limit. To see this, suppose Alice and Bob share the state $1 / \sqrt{2}(|00\rangle+|11\rangle)$, and their measurements take the form of projections onto the states $\cos \frac{\theta_{i}}{2}|0\rangle+\sin \frac{\theta_{i}}{2}|1\rangle$ and $\sin \frac{\theta_{i}}{2}|0\rangle-\cos \frac{\theta_{i}}{2}|1\rangle$, where $\theta_{i}=\frac{i \pi}{2 N}($ Alice's measurements take $i=a$, and Bob's take $i=b$ ). Using this setup, the probability that Alice and Bob's measurement outcomes $X$ and $Y$ are distinct, for $|a-b|=1$, is given by

$$
\sum_{x} P_{X Y \mid A=a, B=b}(x, \bar{x})=\sin ^{2} \frac{\pi}{4 N}
$$

and, likewise, the probability that the outcomes are equal for $a=0$ and $b=2 N-1$ is

$$
\sum_{x} P_{X Y \mid A=0, B=2 N-1}(x, x)=\sin ^{2} \frac{\pi}{4 N} .
$$

Thus, quantum mechanics predicts

$$
I_{N}^{\mathrm{QM}}=2 N \sin ^{2} \frac{\pi}{4 N}
$$

which, in the limit of large $N$, is approximated by $\frac{\pi^{2}}{8 N}$ and can be made arbitrarily small.

\section{TECHNICAL RESULT}

Our argument is based on a straightforward extension of a result about non-signaling distributions $P_{X Y \mid A B}$ by Barrett, Kent, and Pironio [1]. The main difference between their result and our Theorem 1 is that our statement holds with respect to an additional third party with an input $C$ and output $Z$. (When applying the theorem, the local hidden variables will take the place of $Z$, whereas $C$ is not used.)

For the following, let $X$ and $Y$ be binary, $A \in\{0,2, \ldots, 2 N-2\}, B \in\{1,3, \ldots, 2 N-1\}$ for some $N \in \mathbb{N}$, as in Section III and let $Z$ and $C$ be arbitrary.

Theorem 1. If $P_{X Y Z \mid A B C}$ is non-signaling then, for any $C$ chosen independently of the inputs $A$ and $B,{ }^{3}$

$$
D\left(P_{X Z C \mid A=a}, \mathcal{U}_{X} \times P_{Z C}\right) \leq \frac{I_{N}}{2} \quad \text { and } \quad D\left(P_{Y Z C \mid B=b}, \mathcal{U}_{Y} \times P_{Z C}\right) \leq \frac{I_{N}}{2}
$$

for all $a, b$, where $I_{N} \equiv I_{N}\left(P_{X Y \mid A B}\right)$, and where $\mathcal{U}_{X}$ and $\mathcal{U}_{Y}$ denote the uniform distributions on $X$ and $Y$, respectively.

\footnotetext{
${ }^{3}$ Because $C$ is chosen independently of $A$ and $B$, the joint distribution $P_{X Y Z C \mid A B}$ is given by $P_{X Y Z C \mid A=a, B=b}(x, y, z, c)=$ $P_{X Y Z \mid A=a, B=b, C=c}(x, y, z) P_{C}(c)$.
} 
Proof. Using Lemma 1 and the triangle inequality, we have for any fixed $z$ and $c$

$$
\begin{aligned}
& I_{N}\left(P_{X Y \mid A B, C=c, Z=z}\right)=\sum_{\substack{a, b \\
|a-b|=1}} \sum_{x} P_{X Y \mid A=a, B=b, C=c, Z=z}(x, \bar{x})+\sum_{x} P_{X Y \mid A=0, B=2 N-1, C=c, Z=z}(x, x) \\
& \geq \sum_{\substack{a, b \\
|a-b|=1}} D\left(P_{X \mid A=a, C=c, Z=z}, P_{Y \mid B=b, C=c, Z=z}\right)+D\left(P_{\bar{X} \mid A=0, C=c, Z=z}, P_{Y \mid B=2 N-1, C=c, Z=z}\right) \\
& \geq D\left(P_{\bar{X} \mid A=0, C=c, Z=z}, P_{X \mid A=0, C=c, Z=z}\right) .
\end{aligned}
$$

Then, since

$$
D\left(P_{\bar{X} \mid A=0, C=c, Z=z}, P_{X \mid A=0, C=c, Z=z}\right)=2 D\left(P_{X \mid A=0, C=c, Z=z}, \mathcal{U}_{X}\right),
$$

we obtain

$$
D\left(P_{X \mid A=0, C=c, Z=z}, \mathcal{U}_{X}\right) \leq \frac{1}{2} I_{N}\left(P_{X Y \mid A B, C=c, Z=z}\right)
$$

Taking the average over $z$ and $c$ (distributed according to $P_{Z C} \equiv P_{Z \mid C} P_{C}$ ) on both sides of this inequality and using (5) we conclude

$$
D\left(P_{X Z C \mid A=0}, \mathcal{U}_{X} \times P_{Z C}\right) \leq \frac{I_{N}}{2}
$$

The claim for arbitrary $a$ (rather than $a=0$ ) as well as the second inequality of the theorem follow by symmetry.

For our argument, we apply the theorem to the setup described in Section III, with $Z:=(U, V)$ and $C$ equal to a constant (i.e., $C$ is not used). Under the assumption that the hidden variables $U$ and $V$ are independent of the inputs $a$ and $b,{ }^{4}$ we have $P_{Z \mid A=a, B=b} \equiv P_{Z}$. This together with (1) and (2) implies the non-signaling condition. Theorem 1 thus gives

$$
D\left(P_{X U \mid A=a}, \mathcal{U}_{X} \times P_{U}\right) \leq \frac{I_{N}}{2} \quad \text { and } \quad D\left(P_{Y V \mid B=b}, \mathcal{U}_{Y} \times P_{V}\right) \leq \frac{I_{N}}{2}
$$

for all $a$ and $b$. In particular, for $I_{N} \ll 1$, the bound implies that the measurement outcomes $X$ and $Y$ are virtually independent of the local hidden variables $U$ and $V$.

\section{IMPLICATIONS}

Before summarizing the implications of Theorem 1, we first stress that the contribution of this work is not a technical one. Our aim is to establish a connection between an argument proposed in [1] and recent work on hidden variable models, in particular Leggett-type models [6, 7, 8, [9, 10].

Suppose an experiment is performed, using the setup described in Section III. which allows us to estimate an upper bound $I_{N}^{*}$ on the quantity $I_{N} \equiv I_{N}\left(P_{X Y \mid A B}\right)$ defined by (17). Then, according to (12), the maximum locality of $X$, which we measure in terms of its dependence on the local hidden variable $U$ via $D\left(P_{X U \mid A=a}, \mathcal{U}_{X} \times P_{U}\right)$, is bounded by $I_{N}^{*} / 2$.

For example, after many (noiseless) measurements of the $\mathrm{CHSH}$ quantity, $I_{4}$, one would eventually get an upper bound $I_{4}^{*}$ close to $I_{4}^{\mathrm{QM}}=2-\sqrt{2}$ (see Eqn. (8) ). Hence, the maximum locality of a hidden variable theory compatible with these measurements is $1-1 / \sqrt{2} \approx 0.3$. This bound can be brought closer to zero by performing experiments according to the setup described in Section III with larger $N .^{5}$ Such experiments were proposed in [1].

\footnotetext{
4 This assumption simply says that, in an experiment, the choice of measurements $a$ and $b$ must not depend on the value of the local hidden variables. Of course, this is the case if the measurements are chosen at random.

${ }^{5}$ For any given practical setup, the optimal value of $N$ which minimizes the upper bound $I_{N}^{*}$ may depend on the specific noise model of the measurement devices.
} 
In the limit of large $N$, quantum mechanics predicts $I_{\infty}^{\mathrm{QM}}=0$. Hence, for any hidden variable model to describe these quantum correlations, we require $P_{X U \mid A=a}=\mathcal{U}_{X} \times P_{U}$, and $P_{Y V \mid B=b}=\mathcal{U}_{Y} \times P_{V}$. Consequently, the outcomes $X$ and $Y$ for any fixed pair of measurements $(a, b)$ are fully independent of the local hidden variables $U$ and $V$. Notice that, we can reach this conclusion using only measurements in one plane of the Bloch sphere on each side (where Alice's plane contains $a$ and Bob's $b$ ).

Finally, we discuss the implications for Leggett's model. Using our inequality (12) with $N$ measurements in one plane of the Bloch sphere we conclude in the limit of large $N$ that the model can only be consistent with the predictions of quantum mechanics if $\vec{U}$ and $\vec{V}$ are almost orthogonal to the measurement plane. Hence, with measurements in only one plane, we can establish that the local hidden variables $\vec{U}$ and $\vec{V}$ play no rôle. A further advantage of the inequality we use over those of the Leggett-type is that our inequalities enable a device independent falsification of any hidden variable model with non-trivial local part. Conversely, with the usual Leggett-type inequalities, the bound depends on the setup, and is hence less experimentally robust.

[1] J. Barrett, A. Kent, and S. Pironio, Physical Review Letters 97, 170409 (2006).

[2] J. S. Bell, Speakable and unspeakable in quantum mechanics (Cambridge University Press, 1987).

[3] A. Aspect, Nature 398, 189 (1999).

[4] J. Barrett, D. Collins, L. Hardy, A. Kent, and S. Popescu, Physical Review A 66, 042111 (2002).

[5] A. Kent, Physical Review A 72, 012107 (2005).

[6] A. J. Leggett, Foundations of Physics 33, 1469 (2003).

[7] S. Gröblacher, T. Paterek, R. Kaltenbaek, Č. Brukner, M. Żukowski, M. Aspelmeyer, and A. Zeilinger, Nature 446, 871 (2007).

[8] C. Branciard, A. Ling, N. Gisin, C. Kurtsiefer, A. Lamas-Linares, and V. Scarani, Physical Review Letters 99, 210407 (2007).

[9] T. Paterek, A. Fedrizzi, S. Gröblacher, T. Jennewein, M. Żukowski, M. Aspelmeyer, and A. Zeilinger, Physical Review Letters 99, 210406 (2007).

[10] C. Branciard, N. Brunner, N. Gisin, C. Kurtsiefer, A. Lamas-Linares, A. Ling, and V. Scarani, e-print arXiv:0801.2241 (2008).

[11] P. M. Pearle, Phys. Rev. D 2, 1418 (1970).

[12] S. L. Braunstein and C. M. Caves, Annals of Physics 202, 22 (1990).

[13] J. F. Clauser, M. A. Horne, A. Shimony, and R. A. Holt, Physical Review Letters 23, 880 (1969). 